\title{
Inhibition of Polymer Photodegradation by Incorporation of Coffee Silverskin ${ }^{\dagger}$
}

\author{
Aleksander Hejna ${ }^{1, *}$, Mateusz Barczewski ${ }^{2}$, Paulina Kosmela ${ }^{1}$ and Olga Mysiukiewicz ${ }^{2}$ \\ 1 Department of Polymer Technology, Gdańsk University of Technology, Narutowicza 11/12 80-233 Gdańsk, \\ Poland; paulina.kosmela@pg.edu.pl \\ 2 Institute of Materials Technology, Poznan University of Technology, Piotrowo 3, 61-138 Poznań, Poland; \\ mateusz.barczewski@put.poznan.pl (M.B.), olga.mysiukiewicz@put.poznan.pl (O.M.) \\ * Correspondence: aleksander.hejna@pg.gda.pl; Tel.: +48-58-347-2234 \\ + Presented at the First International Conference on “Green" Polymer Materials 2020, 5-25 November 2020; \\ Available online: https://cgpm2020.sciforum.net/.
}

Published: 4 November 2020

\begin{abstract}
Over the last years, the trend associated with the incorporation of materials from renewable resources into polymer technology is getting significantly more vital. Researchers are trying to transfer the properties of natural raw materials into the polymer world. Therefore, different natural materials are more often investigated as potential additives for polymers. Such an effect is noted for the coffee industry by-products, such as coffee silverskin. Because of the relatively high contents of compounds showing antioxidant activity, such as caffeine, polyphenols, tannins, or melanoidins, this by-product could be considered not only as a filler, but also as a potential modifier for polymer materials. Its antioxidant activity is comparable to commercially available antioxidants applied in polymer technology. Therefore, in the presented paper, we examined the influence of the coffee silverskin (from 1 to $20 \mathrm{wt} \%$ ) on the thermal and mechanical performance of polyethylenebased composites. Moreover, materials were subjected to accelerated aging tests in the UV chamber, which revealed that coffee silverskin could inhibit the photodegradation of the polymer matrix. Therefore, this by-product should be considered as an exciting alternative for the conventional lignocellulosic fillers, which could provide additional features to polymer composites.
\end{abstract}

Keywords: coffee silverskin; antioxidant activity; accelerated aging; polymer composites; mechanical properties;

\section{Introduction}

Coffee silverskin is one of the by-products generated by the coffee industry. It is not the most problematic one, because it stands only for $\sim 4.2 \mathrm{wt} \%$ of coffee beans, so it is not generated in massive amounts [1]. Moreover, it is generated during roasting of coffee, performed at relatively high temperatures, resulting in the very low moisture content, which makes it very interesting for the potential industrial applications. The chemical composition of coffee silverskin is relatively similar to the materials conventionally applied as fillers for wood-polymer composites. It usually contains between 60 and $75 \%$ of fiber, mostly insoluble, including up to $30 \%$ of lignin [2]. Coffee silverskin also contains $15-19 \%$ of proteins, which can provide additional features to the polymer composites [3]. They may act as plasticizers, but due to the presence of additional functional groups may also provide additional possibilities for interfacial interactions with polar polymer matrices.

Nevertheless, the most interesting is the content of compounds showing antioxidant activity, such as caffeine, polyphenols, tannins, or melanoidins generated during roasting of coffee [4]. Generally, silverskin is considered as an excellent source of these compounds, however, their content, and to be more precise, the yield of their recovery strongly depends on the extraction method or 
selected solvents [5]. Therefore, reported values of the total phenolics content varied from 2.3 to even $36.0 \mathrm{mg}$ gallic acid/g, resulting in the antioxidant activity, determined by the (2,2-diphenyl-1-picrylhydrazyl-hydrate) (DPPH) assay, in the range of 9.21-18.24 $\mu \mathrm{mol} \mathrm{TR} / \mathrm{g}$ [6]. The main compounds showing antioxidant activity present in coffee silverskin are caffeine, epicatechin, as well as caffeic, caffeoylquinic, $p$-coumaric, $p$-coumaroylquinic, ferulic, feruloylquinic, gallic, syringic, transcynnamic, and vanillic acids [7]. Other, less popular include hyperoside, kaempferol, naringin, quercetin, or quinine.

The compounds mentioned above may potentially act as antioxidants for various polymer matrices. Therefore, the presented paper aimed to investigate the impact of the coffee silverskin content (from 1 to $20 \mathrm{wt} \%$ ) on the thermal and mechanical properties of polyethylene-based composites. The performance of the composites was also analyzed after accelerated aging tests in the UV chamber to determine the efficiency of the coffee silverskin as a potential antioxidant for polymer composites.

\section{Experimental}

\subsection{Materials}

High-density polyethylene (HDPE), type M300054, obtained from SABIC (The Netherlands), was applied as a matrix for the preparation of investigated composites. According to producer data, its density equaled $0.954 \mathrm{~g} / \mathrm{cm}^{3}$, and it was characterized by a melt flow rate of $30 \mathrm{~g} / 10 \mathrm{~min}\left(190{ }^{\circ} \mathrm{C}\right.$, $2.16 \mathrm{~kg}$ ). Coffee silverskin was obtained from the local coffee roastery.

\subsection{Preparation of polymer composites}

The composites were prepared by mixing in a molten state. To facilitate the mixing process, the HDPE pellets were pulverized into a fine powder using a Tria 25-16/TC-SL high-speed knife grinder. Then the polymeric powder was preliminary mixed with $1,2,5,10$, or $20 \mathrm{wt} \%$ of coffee silverskin. Preliminary to mixing in molten state compositions were dried in laboratory cabined dryer Memmert ULE 500 for $12 \mathrm{~h}$ at $70^{\circ} \mathrm{C}$. The mixtures were processed using a ZAMAK EH16.2D co-rotating twinscrew extruder operating at $100 \mathrm{rpm}$ with the maximum temperature of the process of $190{ }^{\circ} \mathrm{C}$. The obtained materials were cooled in forced airflow and pelletized. The resulting composites were then compression molded at $170{ }^{\circ} \mathrm{C}$ and $4.9 \mathrm{MPa}$ for $2 \mathrm{~min}$ and then kept under pressure at room temperature for another $5 \mathrm{~min}$ to enable solidification of the material. The unfilled HDPE was processed along with its composites. The specimens were named in reference to their filler content, as $\mathrm{PE}$ and $\mathrm{PE} / \mathrm{X} \mathrm{KK}$, where $\mathrm{X}$ stands for the filler content.

\subsection{Accelerated aging of composites}

After 7-day conditioning at room temperature, samples were subjected to the accelerated aging process with the use of using TestAn Xentest 2200 xenon light accelerated aging chamber. The aging process was conducted under $0.55 \mathrm{~W} / \mathrm{m}^{2}$ irradiance at $340 \mathrm{~nm}$ UV light intensity, with constant max. temperature up to $63{ }^{\circ} \mathrm{C}$ during dry UV light exposure. The samples were subjected to UV light exposure in two time periods of 100 and $200 \mathrm{~h}$, which was used for sample description in the further part of the manuscript.

\subsection{Measurements}

Oxidation induction time (OIT) of analyzed composites was determined by the differential scanning calorimetry (DSC) analysis. The $5 \mathrm{mg}$ samples were placed in aluminum crucibles with pierced lids. They were heated from 20 to $190{ }^{\circ} \mathrm{C}$ with a heating rate of $20^{\circ} \mathrm{C} / \mathrm{min}$ in nitrogen, then kept at $190{ }^{\circ} \mathrm{C}$ for $5 \mathrm{~min}$ in nitrogen, and then gas was switched to oxygen and the time required for sample oxidation was measured. The measurements were conducted using a Netzsch 204F1 Phoenix apparatus. 
To determine the crystallization and melting temperatures, as well as the crystalline structure of analyzed composites, DSC analysis was applied. The $5 \mathrm{mg}$ samples were placed in aluminum crucibles with pierced lids. They were heated from 20 to $250{ }^{\circ} \mathrm{C}$ with a heating rate of $10^{\circ} \mathrm{C} / \mathrm{min}$ and then cooled back to the initial temperature with a cooling rate of $10^{\circ} \mathrm{C} / \mathrm{min}$. The heating/cooling cycle was performed twice in order to erase the thermal history of the polymers during the first heating. The measurements were conducted using a Netzsch 204F1 Phoenix apparatus, in an inert atmosphere of nitrogen. The crystallinity degree $X_{c r}$ of the samples was calculated using formula (1):

$$
X_{c r}=\left(\Delta H_{m} /\left((1-\varphi) \cdot \Delta H_{m 100 \%}\right)\right) \cdot 100 \%
$$

where: $\Delta H_{m}$ - melting enthalpy of a sample, $\Delta H_{m 100 \%}$ - melting enthalpy of $100 \%$ crystalline polyethylene, $\Delta H_{m 100 \%}=293.6 \mathrm{~J} / \mathrm{g}[8], \varphi-$ filler weight fraction.

The tensile strength and elongation at break were estimated following ISO 527 for dumbbell samples type 5B. Tensile tests were performed on a Zwick/Roell Z020 apparatus with a cell load capacity of $20 \mathrm{kN}$ at a constant speed of $5 \mathrm{~mm} / \mathrm{min}$.

\section{Results}

\subsection{Thermal properties}

To determine the potential antioxidant activity of coffee silverskin, and its effect on the thermooxidative stability of prepared composites, the oxidation induction time was determined by DSC analysis. In Figure 1 there are presented thermograms for unaged samples, as well as for PE and $\mathrm{PE} / 20 \mathrm{EK}$ depending on aging time. Moreover, in Table 1 there are presented values of oxidation induction time for all samples, along with the results of "conventional" DSC analysis. It can be seen that the introduction of coffee silverskin caused a significant delay in composites' oxidation process, confirming the antioxidant activity of this by-product. Values of OIT were over 2.5 times bigger even for $1 \mathrm{wt} \%$ content of filler, comparing to $20 \mathrm{~min}$ for neat polyethylene matrix. For higher contents OIT values reached even 130-140 min. Above $5 \mathrm{wt} \%$ the effect was not as distinct. Accelerated aging of samples significantly reduced the thermooxidative stability of analyzed composites. Nevertheless, sample PE/20ŁK still showed higher stability than neat PE, even after 200 hours of aging, which points to the very high activity of antioxidant compounds present in coffee silverskin. In Figure $1 \mathrm{~b}$ it can also be seen that magnitude of signals was significantly reduced after aging, indicating partial oxidation of the material.
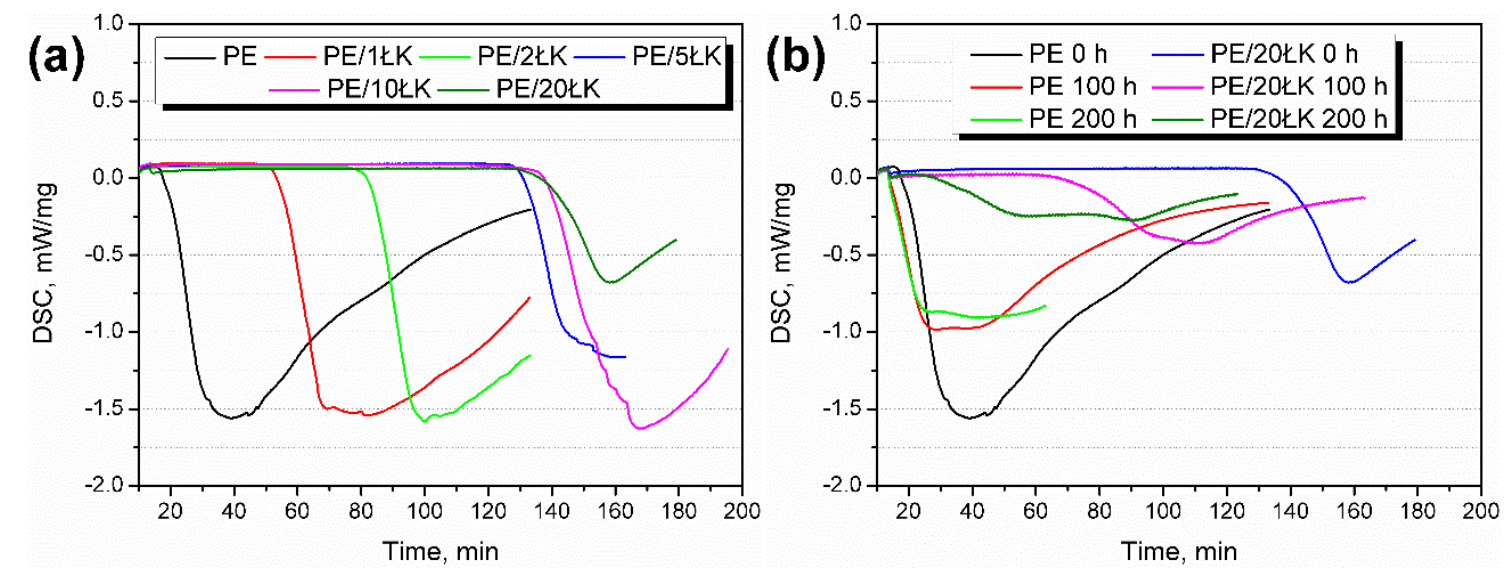

Figure 1. Thermograms of (a) unaged composite samples, and (b) PE and PE/20€K composites depending on aging time.

Incorporation of the coffee silverskin into the polyethylene matrix did not substantially affect the thermal properties of unaged samples. Temperatures of melting and crystallization were hardly affected by the filler incorporation. The decrease of the melting enthalpy $(\Delta \mathrm{Hm})$ was noted, which was associated with the reduced share of the polymer matrix. Nevertheless, the degree of crystallinity was slightly increased from 63.8 to $66.6 \%$, which was related to the nucleating activity of filler. This 
phenomenon can be related to the previously described cases of the interaction of lignocellulosic fillers as heterogeneous nucleants of the PE [9].

Aging of samples did not cause noticeable changes in the $T_{m}$ and $T_{c r}$ values, but affected the melting enthalpy and degree of crystallinity, especially for unmodified polyethylene and sample PE/1ŁK. After $200 \mathrm{~h}$ of aging, the degree of crystallinity for this samples was significantly reduced, pointing to the partial decomposition of the material.

Table 1. Results of DSC analysis for prepared composites.

\begin{tabular}{|c|c|c|c|c|c|c|}
\hline Sample & Aging time, $\mathrm{h}$ & $\mathrm{T}_{\mathrm{m}},{ }^{\circ} \mathrm{C}$ & $\mathrm{T}_{\mathrm{cr}},{ }^{\circ} \mathrm{C}$ & $\Delta \mathrm{H}_{\mathrm{m}}, \mathrm{J} / \mathrm{g}$ & $\mathrm{X}_{\mathrm{cr},} \%$ & OIT, min \\
\hline \multirow{3}{*}{ PE } & 0 & 134.1 & 112.8 & 187.4 & 63.8 & 20.0 \\
\hline & 100 & 132.5 & 112.4 & 197.8 & 67.4 & 13.5 \\
\hline & 200 & 133.2 & 110.6 & 146.6 & 49.9 & 13.5 \\
\hline \multirow{3}{*}{ PE/1ŁK } & 0 & 133.2 & 113.1 & 190.5 & 65.5 & 54.8 \\
\hline & 100 & 132.7 & 112.3 & 189.1 & 65.1 & 13.5 \\
\hline & 200 & 133.8 & 113.5 & 152.1 & 52.3 & 13.5 \\
\hline \multirow{3}{*}{ PE/2ŁK } & 0 & 134.1 & 113.0 & 188.9 & 65.7 & 83.9 \\
\hline & 100 & 132.8 & 112.9 & 181.8 & 63.2 & 15.1 \\
\hline & 200 & 132.7 & 112.8 & 182.1 & 63.3 & 13.5 \\
\hline \multirow{3}{*}{ PE/5ŁK } & 0 & 133.3 & 113.3 & 186.5 & 66.9 & 131.9 \\
\hline & 100 & 133.6 & 113.1 & 174.8 & 62.7 & 15.8 \\
\hline & 200 & 132.0 & 113.8 & 177.9 & 63.8 & 16.5 \\
\hline \multirow{3}{*}{ PE/10€K } & 0 & 133.0 & 113.4 & 174.5 & 66.0 & 139.5 \\
\hline & 100 & 132.3 & 112.7 & 170.9 & 64.7 & 17.9 \\
\hline & 200 & 132.4 & 112.9 & 170.7 & 64.6 & 16.7 \\
\hline \multirow{3}{*}{ PE/20ŁK } & 0 & 132.6 & 112.5 & 156.4 & 66.6 & 140.4 \\
\hline & 100 & 133.6 & 112.2 & 148.5 & 63.2 & 76.8 \\
\hline & 200 & 131.6 & 113.6 & 159.2 & 67.8 & 30.1 \\
\hline
\end{tabular}

\subsection{Mechanical properties}

In Figure 2 there are presented tensile properties of the analyzed PE/ŁK composites before and after the accelerated aging. The initial values of tensile strength were at a similar level for neat polyethylene matrix and composites containing up to $5 \mathrm{wt} \%$ of coffee silverskin. For higher loadings of filler, drop of the tensile strength was noted. Similar effects were observed in our other work [10]. Such a phenomenon is associated with the insufficient interfacial adhesion between filler and matrix, which was especially pronounced for the elongation at break. This parameter was drastically decreased when solid particles were introduced into the polymer matrix.

The aging of composite samples resulted in noticeable changes in their mechanical performance. Nevertheless, a very beneficial influence of coffee silverskin can be noted. For the neat polyethylene matrix, a drop of tensile strength, and especially elongation at break was very significant after aging. Similar effects were noted by other researchers [11]. It can be seen that the incorporation of even 1 $w t \%$ of coffee silverskin caused a noticeable improvement of the mechanical performance. The share of the initial values of mechanical properties was increasing with the loading of coffee silverskin. It confirms the results of the thermal properties analysis indicating the elongation of oxidation induction time with the addition of applied coffee industry by-product. 

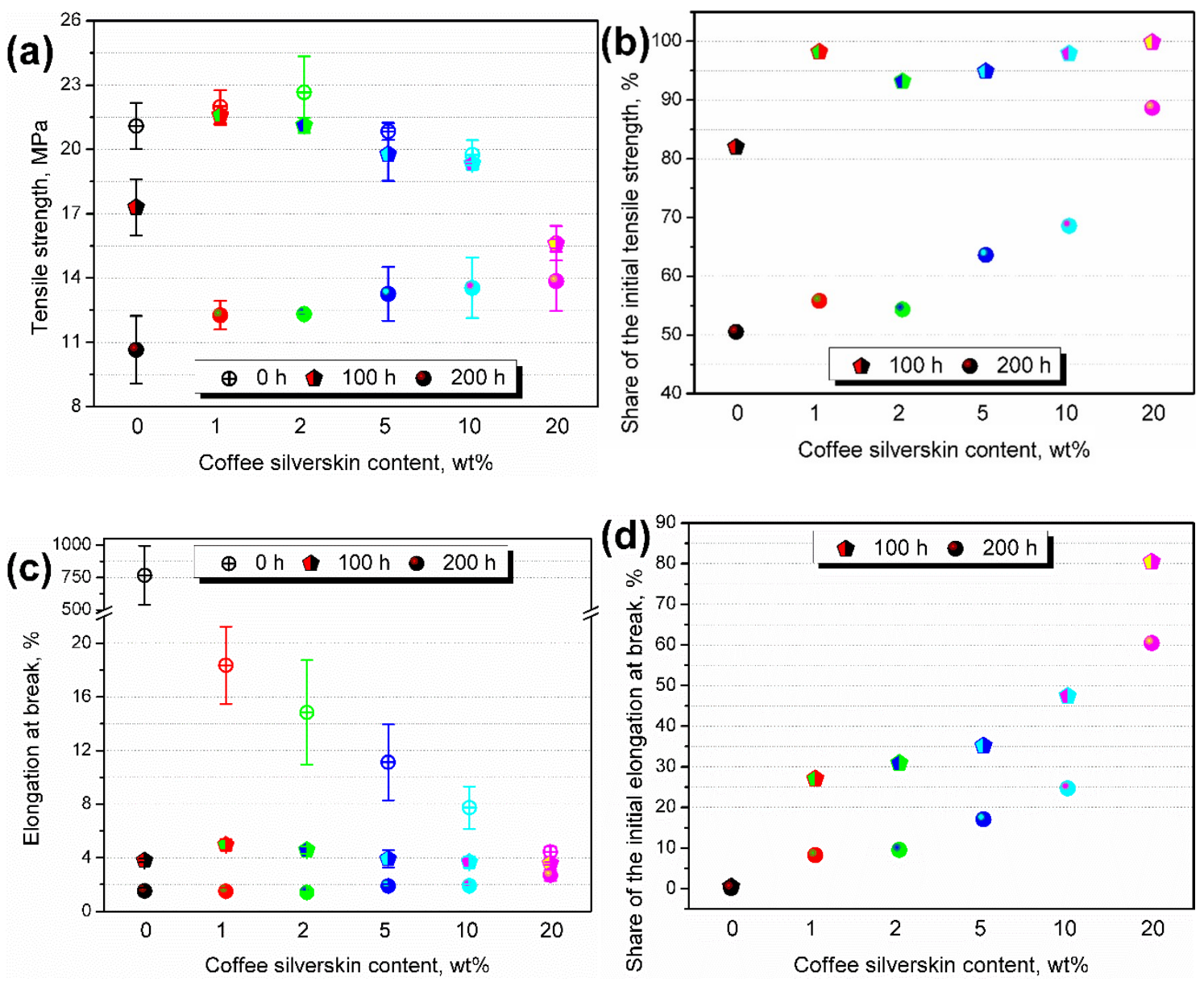

Figure 2. Plots showing (a) tensile strength, (b) share of the initial tensile strength, (c) elongation at break, and (d) share of the initial elongation at break for PE/ŁK composites depending on the filler content.

\section{Conclusions}

In the presented work, the polyethylene-based composites modified with natural waste filler in the form of coffee silverskin were produced and tested. It has been shown that incorporated filler shows novel functionality, in the form of an antioxidant effect, resulting from the active compounds it contains. The introduction of a lignocellulosic filler with a complex structure and chemical composition made it possible to suppress the aging effects caused by exposure to UV radiation in controlled conditions. Thermal analysis, performed by differential scanning calorimetry, was correlated to and remained in agreement with the results of mechanical studies performed using tensile test evaluation. Based on the obtained results, it can be concluded that coffee silverskin can be used as a low-cost filler for the production of highly-filled composites with additional functionality in comparison to different lignocellulosic waste fillers, as well as an effective additive which influences negative effects of oxidation induced by aging processes.

Author Contributions: A.H. and M.B. conceived and designed the experiments; A.H., M.B., P.K. and O.M. performed the experiments; A.H., M.B., P.K. and O.M. analyzed the data; A.H. and M.B. contributed reagents/materials/analysis tools; A.H., M.B., P.K. and O.M. wrote the paper.

Conflicts of Interest: The authors declare no conflict of interest.

\section{References}

1. Esquivel, P.; Jiménez, V.M. Functional properties of coffee and coffee by-products. Food Res. Int. 2012, 46, 488-495, https://doi.org/10.1016/j.foodres.2011.05.028. 
2. Janissen, B.; Huynh, T. Chemical composition and value-adding applications of coffee industry byproducts: A review. Resour. Conserv. Recy. 2018, 128, 110-117, https://doi.org/10.1016/j.resconrec.2017.10.001.

3. Ballesteros, L.F.; Teixeira, J.A.; Mussatto, S.I. Chemical, Functional, and Structural Properties of Spent Coffee Grounds and Coffee Silverskin. Food Bioprocess Tech. 2014, 7, 3493-3503, https://doi.org/10.1007/s11947-014-1349-z.

4. Barbosa-Pereira, L.; Guglielmetti, A.; Zeppa, G. Pulsed Electric Field Assisted Extraction of Bioactive Compounds from Cocoa Bean Shell and Coffee Silverskin. Food Bioprocess Tech. 2018, 11, 818-835, https://doi.org/10.1007/s11947-017-2045-6.

5. Ballesteros, L.F.; Teixeira, J.A.; Mussatto, S.I. Selection of the Solvent and Extraction Conditions for Maximum Recovery of Antioxidant Phenolic Compounds from Coffee Silverskin. Food Bioprocess Tech. 2013, 7, 1322-1332, https://doi.org/10.1007/s11947-013-1115-7.

6. Conde, T.; Mussatto, S.I. Isolation of polyphenols from spent coffee grounds and silverskin by mild hydrothermal pretreatment. Prep. Biochem. Biotech. 2015, 46, 406-409, https://doi.org/10.1080/10826068.2015.1084514.

7. Bresciani, L.; Calani, L.; Bruni, R.; Brighenti, F.; Del Rio, D. Phenolic composition, caffeine content and antioxidant capacity of coffee silverskin. Food Res. Int. 2014, 61, 196-201, https://doi.org/10.1016/j.foodres.2013.10.047.

8. Kodjie, S.L.; Li, L.; Li, B.; Cai, W.; Li, C.Y.; Keating, M. Morphology and crystallization behavior of HDPE/CNT nanocomposite. J. Macromol. Sci. Phys. 2006, 45, 231-245, https://doi.org/10.1080/00222340500522299.

9. Andrzejewski, J.; Krawczak, A.; Wesoły, K.; Szostak, M. Rotational molding of biocomposites with addition of buckwheat husk filler. Structure-property correlation assessment for materials based on polyethylene (PE) and poly(lactic acid) PLA. Compos. Part B-Eng. 2020, 108410, https://doi.org/10.1016/j.compositesb.2020.108410.

10. Mysiukiewicz, O.; Kosmela, P.; Barczewski, M.; Hejna, A. Mechanical, Thermal and Rheological Properties of Polyethylene-Based Composites Filled with Micrometric Aluminum Powder. Materials 2020, 13, 1242, https://doi.org/10.3390/ma13051242.

11. Méndez-Hernández, M.L.; Tena-Salcido, C.S.; Sandoval-Arellano, Z.; González-Cantú, M.C.; Mondragón, M.; Rodríguez-González, F.J. The effect of thermoplastic starch on the properties of HDPE/TPS blends during UV-accelerated aging. Polym. Bull. 2011, 67, 903-914, https://doi.org/10.1007/s00289-011-0501-4.

(C) 2020 by the authors. Submitted for possible open access publication under the terms and conditions of the Creative Commons Attribution (CC BY) license (http://creativecommons.org/licenses/by/4.0/). 